\title{
Demonstration of three-port grating phase relations
}

\author{
A. Bunkowski, O. Burmeister, K. Danzmann, and R. Schnabel \\ Albert-Einstein-Institut Hannover, Max-Planck-Institut für Gravitationsphysik and Universität Hannover, \\ Callinstrasse 38, 30167 Hannover, Germany
}

T. Clausnitzer, E.-B. Kley, and A. Tünnermann

Institut für Angewandte Physik, Friedrich-Schiller-Universität Jena, Max-Wien-Platz 1, 07743 Jena, Germany

Received April 19, 2006; accepted May 24, 2006;

posted May 31, 2006 (Doc. ID 69970); published July 25, 2006

\begin{abstract}
We experimentally demonstrate the phase relations of three-port gratings by investigating three-port coupled Fabry-Perot cavities. Two different gratings that have the same first-order diffraction efficiency but differ substantially in their second-order diffraction efficiency have been designed and manufactured. Using the gratings as couplers to Fabry-Perot cavities, we could validate the results of an earlier theoretical description of the phases at a three-port grating [Opt. Lett. 30, 1183 (2005)] () 2006 Optical Society of America OCIS codes: $050.5080,120.2230,230.1360$.
\end{abstract}

Conventional interferometers rely on splitting and recombining optical fields with partly transmissive beam splitters. When transmission through optical substrates is disadvantageous, diffractive reflection gratings can also serve as beam splitters, allowing for all-reflective interferometry. ${ }^{1}$ As long as the grating splits an incoming beam into two outgoing beams, the phase relation at the grating, and hence the properties of the interferometer built thereof, are analogous to the well-known ones of a transmissive twoport beam splitter. However, if a diffractive beam splitter has more than two orders, the mirror analog, and thus the simple phase relation, no longer hold. Still, a knowledge of these relations at the diffractive beam splitter is essential for an understanding of multiple-port interferometry. In a recent experiment, a grating in a second-order Littrow mount was used to couple light into a Fabry-Perot cavity. ${ }^{2}$ In this case, the incoming beam was split into three outgoing beams. The phase relations at the so-called threeport grating were analyzed theoretically and the input-output relations for a Fabry-Perot cavity with a three-port coupler were derived. ${ }^{3}$ The theoretical investigation of the phases was based solely on energy conservation and reciprocity of the device, but there has not yet been an experimental validation of the results.

In this Letter we report an experiment that was performed to demonstrate the phase relations of optical three-port devices. Two different gratings were designed and manufactured for this purpose, and used as couplers to Fabry-Perot interferometers.

Phase relations for three-port gratings with equal diffraction efficiencies in the \pm first orders can be written $\operatorname{as}^{3,4}$

$$
\begin{aligned}
& \phi_{0}=0, \\
& \phi_{1}=-(1 / 2) \arccos \left[\left(\eta_{1}^{2}-2 \eta_{0}^{2}\right) /\left(2 \rho_{0} \eta_{0}\right)\right], \\
& \phi_{2}=\arccos \left[-\eta_{1}^{2} /\left(2 \eta_{2} \eta_{0}\right)\right],
\end{aligned}
$$

where $\phi_{0}, \phi_{1}$, and $\phi_{2}$ are the phase shifts for zeroth, first, and second-diffraction orders, respectively. Interestingly, the coupling phases depend on the coupling amplitudes, which are given by $\eta_{0}, \eta_{1}$, and $\eta_{2}$ for the zeroth, first, and second-diffraction orders, respectively, and by $\rho_{0}$ for the normal incidence reflectivity of the grating.

Direct measurements of beam-splitter phase relations are difficult. However, if the three-port beam splitter is used to couple light into a cavity, the cavity properties can be used to validate the phase relations. Figure 1 shows the optical layout of a FabryPerot interferometer with a three-port grating coupler. The grating is used in a second-order Littrow mount, and light from a laser source is coupled to the interferometer via the grating's first order. The field amplitudes of the back-reflected light $\left(c_{1}\right)$ and forward-reflected light $\left(c_{3}\right)$ result from interference of the input field with the intracavity field and directly depend on the phase relations between the grating ports. In Ref. 3, amplitude reflection coefficients for $c_{1}$ and $c_{3}$, as well as the amplitudes for the intracavity field $\left(c_{2}\right)$ and the transmitted field $(t)$, were derived and are repeated here for convenience.

$$
\begin{aligned}
& c_{1}=\eta_{2} \exp \left(i \phi_{2}\right)+\eta_{1}^{2} \exp \left[2 i\left(\phi_{1}+\phi\right)\right] d, \\
& c_{2}=\eta_{1} \exp \left(i \phi_{1}\right) d, \\
& c_{3}=\eta_{0}+\eta_{1}^{2} \exp \left[2 i\left(\phi_{1}+\phi\right)\right] d,
\end{aligned}
$$

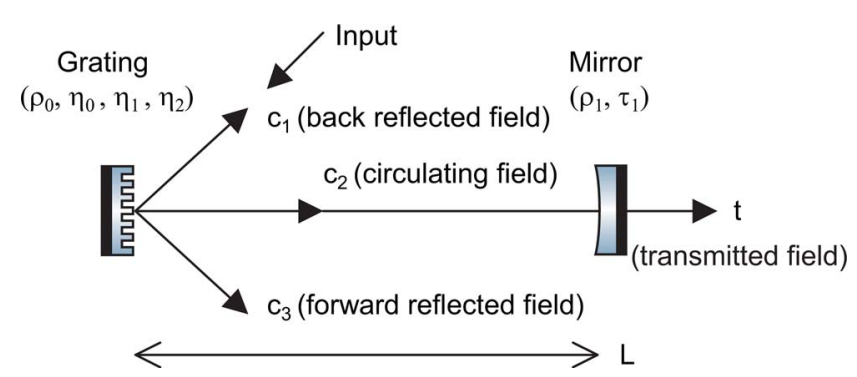

Fig. 1. (Color online) Grating in second-order Littrow mount with naming convention given in text. 


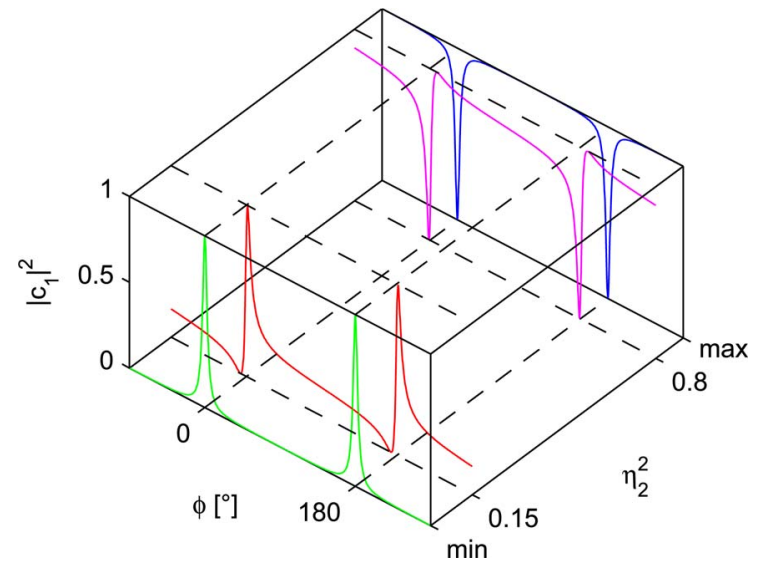

Fig. 2. (Color online) Calculated power back reflectance $\left|c_{1}\right|^{2}$ for a cavity with coupling $\eta_{1}^{2}=0.1$ and an end mirror with $\rho_{1}=1$ as a function of cavity tuning $(\phi)$ for selected values of second-order diffraction efficiency $\eta_{2}^{2}$.

$$
t=i \tau_{1} c_{2} \exp (i \phi)
$$

where the amplitude reflectance and transmittance of the cavity end mirror are given by $\rho_{1}$ and $\tau_{1}$, respectively. The resonance factor is given by $d=[1$ $\left.-\rho_{0} \rho_{1} \exp (2 i \phi)\right]^{-1}$, and the length $L$ of the cavity is expressed by the tuning parameter $\phi=\omega L / c$, where $\omega$ is the angular frequency and $c$ the speed of light.

One distinct feature of this type of grating cavity is that the grating phase relations allow for reflection coefficients (as a function of $\phi$ ) that are not symmetric to the detuning of the cavity. Figure 2 shows the calculated power back reflectance $\left|c_{1}\right|^{2}$ of a cavity with input coupling of $\eta_{1}^{2}=0.1$ and an ideal end mirror $\left(\rho_{1}=1\right)$ as a function of cavity tuning $\phi$ for selected values of the second-order diffraction efficiency $\eta_{2}^{2}$. In all cases shown, the cavity finesse is the same. For an ideal (lossless) grating, the finesse depends on the first-order diffraction efficiency $\eta_{1}=\left[\left(1-\rho_{0}\right) / 2\right]^{1 / 2}$ only. For the minimal second-order diffraction efficiency $^{3} \eta_{2 \text {,min }}=\left(1-\rho_{0}\right) / 2$, all the light is reflected back towards the laser source if the cavity is on resonance $(\phi=0 \bmod \pi)$. However, for maximal secondorder diffraction efficiency $\eta_{2, \max }=\left(1+\rho_{0}\right) / 2$, no light is reflected back from a resonating cavity. Hence for the extremal values of $\eta_{2}$ the back-reflected port behaves either exactly like the reflection port or the transmission port of a conventional, impedancematched, two-mirror Fabry-Perot cavity. For intermediate values of $\eta_{2}$, the power reflectance is no longer symmetric to the $\phi=0$ axis, and the resonance peaks are not of the usual Airy form, as can be seen for the two exemplary curves, $\eta_{2}^{2}=0.15$ and $\eta_{2}^{2}=0.8$, in Fig. 2.

To verify the grating behavior, two gratings with essentially the same first-order diffraction efficiency but substantially different second- and hence zerothorder diffraction efficiency were designed and manufactured. The gratings use a binary structure written into the top layer of a dielectric multilayer stack consisting of $\mathrm{Ta}_{2} \mathrm{O}_{5}$ and $\mathrm{SiO}_{2}$ placed on a fused silica substrate. We chose a grating period of $p=1450 \mathrm{~nm}$, which corresponds to a second-order Littrow angle of $47.2^{\circ}$ for the $\mathrm{Nd}$ :YAG laser wavelength of $1064 \mathrm{~nm}$ used. A rigorous coupled wave analysis ${ }^{5}$ was performed to design the grating. The ridge width is $p / 2$, and the top layer consists of $880 \mathrm{~nm}$ of $\mathrm{SiO}_{2}$. Figure 3 shows the calculated diffraction efficiencies for all three diffraction orders in the second-order Littrow mount as a function of groove depth. The gratings were produced by ultrafast high-accuracy electron beam direct writing ${ }^{6}$ (electron beam writer ZBA23h from Leica Microsystems Jena $\mathrm{GmbH}$ ) and etched by means of reactive ion beam etching. The etching process was stopped after reaching a groove depth of $500 \mathrm{~nm}$ (G1) and $850 \mathrm{~nm}$ (G2), respectively.

A sketch of the experimental setup used to verify the grating phase relations is shown in Fig. 4. A beam of a diode-pumped $\mathrm{Nd}$ :YAG nonplanar ring oscillator (Model Mephisto from Innolight $\mathrm{GmbH}$ ) was spatially filtered with a triangular ring cavity. The grating (either G1 or G2) was illuminated at a secondorder Littrow angle, and a cavity end mirror with $\tau_{1}^{2}$ $=300$ parts per million was placed parallel to the grating's surface. The cavity length could be controlled by a piezoelectric transducer, and the three ports of interest were monitored by photodetectors.

Figures 5 and 6 show the measured signals from the three photodetectors for linear cavity scans over one free spectral range using G1 and G2, respectively. Also shown are the theoretical curves $\left|c_{1}(\phi)\right|^{2}$,

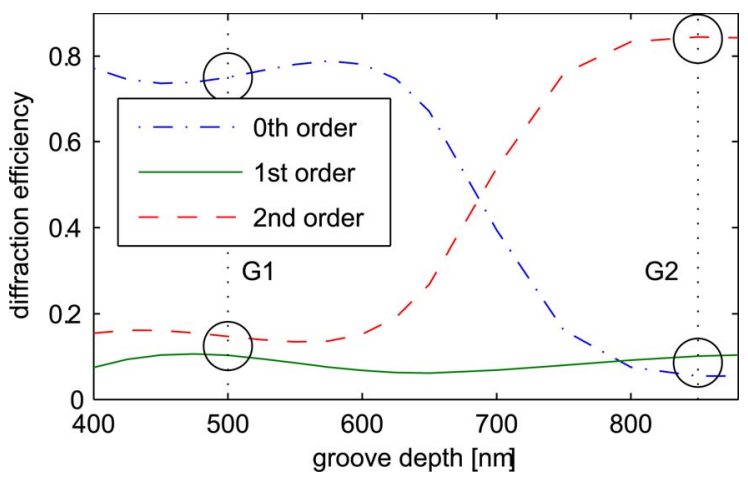

Fig. 3. (Color online) Calculated diffraction efficiencies as a function of groove depth obtained with RCW calculations for the gratings used. The circles show the design values of our gratings $\mathrm{G} 1$ and $\mathrm{G} 2$, respectively.

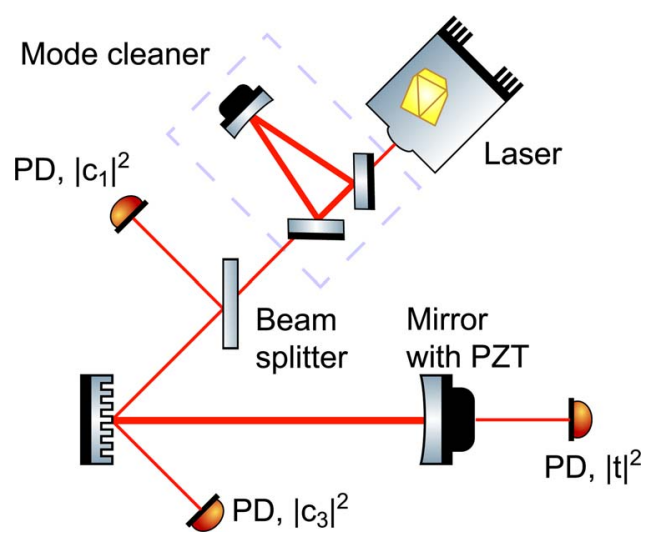

Fig. 4. (Color online) Experimental setup: PZT, piezoelectric transducer; $\mathrm{PD}$, photodetector. 

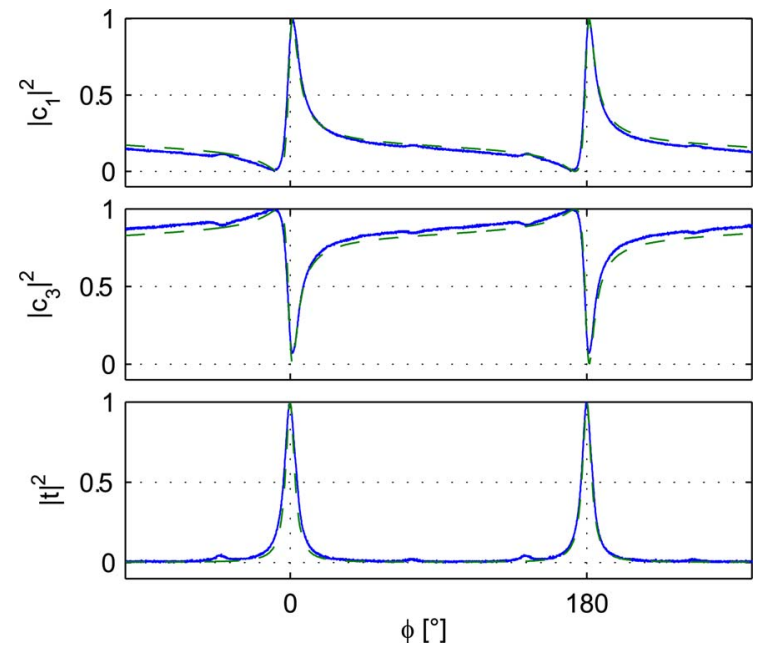

Fig. 5. (Color online) Normalized powers at the three photodetectors for three-port coupler G1 as the cavity length was linearly scanned (solid, blue curve) and the calculated values (dashed-dotted, green curve).

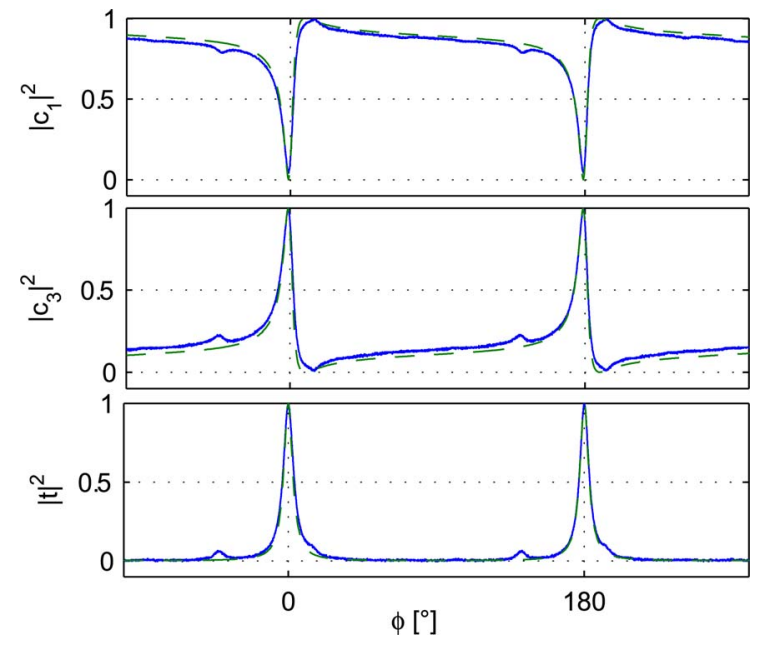

Fig. 6. (Color online) Normalized powers at the three photodetectors for three-port coupler G2 as the cavity length was linearly scanned (solid, blue curve) and the calculated values (dashed, green curve).

$\left|c_{3}(\phi)\right|^{2}$, and $|t(\phi)|^{2}$, which were obtained from Eqs. (4), (6), and (7) using measured efficiencies of the two gratings. Coupling to the cavity was measured to be identical for both gratings within the measurement accuracy of about $5 \%$ of the power meter used, $\eta_{1}^{2}(\mathrm{G} 1)=\eta_{1}^{2}(\mathrm{G} 2)=0.10$. For the first grating, a value of $\eta_{2}^{2}(\mathrm{G} 1)=0.15$ was measured, and for the second one, a value of $\eta_{0}^{2}(\mathrm{G} 2)=0.10$ was measured. The remaining values were calculated using the identities $\eta_{0}^{2}+\eta_{1}^{2}$ $+\eta_{2}^{2}=1$ and $\rho_{0}^{2}+2 \eta_{1}^{2}=1$. We found the calculated values within the error bars of direct measurements.

Figures 5 and 6 show that the theoretical and measured curves agree very well. The interference at the three-port gratings could therefore be well described by the phase relations according to Eqs. (1)-(3). The small deviations are possibly due to imperfect mode matching, and losses at the grating that may be caused by transmission, scattering, and diffraction from periodic grating errors. As predicted, the measured intensities in the reflecting ports showed the asymmetric behavior around cavity resonances.

In conclusion, we have designed and manufactured two diffraction gratings that allowed the construction of grating-coupled Fabry-Perot cavities with the same finesse but with totally different properties of the two reflected ports, thereby confirming the phase relations that were earlier derived theoretically. Our experimental results could be fully described by phase relations based on energy conservation and reciprocity and the knowledge of the grating's diffraction efficiencies. No further information about the gratings was required.

This research was supported by the Deutsche Forschungsgemeinschaft within the Sonderforschungsbereich TR7. A. Bunkowski's e-mail address is alexander.bunkowski@aei.mpg.de.

\section{References}

1. K.-X. Sun and R. L. Byer, Opt. Lett. 23, 567 (1997).

2. A. Bunkowksi, O. Burmeister, P. Beyersdorf, K. Danzmann, R. Schnabel, T. Clausnitzer, E.-B. Kley, and A. Tünnermann, Opt. Lett. 29, 2342 (2004).

3. A. Bunkowski, O. Burmeister, K. Danzmann, and R. Schnabel, Opt. Lett. 30, 1183 (2005).

4. R. Schnabel, A. Bunkowski, O. Burmeister, and K. Danzmann, Opt. Lett. 31, 658 (2006).

5. M. G. Moharam and T. K. Gaylord, J. Opt. Soc. Am. 72, 1385 (1982).

6. E.-B. Kley, T. Clausnitzer, M. Cumme, K. Zöllner, and B. Schnabel, in Proc. SPIE 4231, 116 (2000). 